\title{
Boolean Algebras in Visser Algebras
}

\author{
Majid Alizadeh, Mohammad Ardeshir, and Wim Ruitenburg
}

\begin{abstract}
We generalize the double negation construction of Boolean algebras in Heyting algebras to a double negation construction of the same in Visser algebras (also known as basic algebras). This result allows us to generalize Glivenko's theorem from intuitionistic propositional logic and Heyting algebras to Visser's basic propositional logic and Visser algebras.
\end{abstract}

\section{Introduction}

Basic propositional calculus (BPC), which was introduced by Albert Visser in [8], captures a sublogic of intuitionistic propositional calculus (IPC) which corresponds to modal logic K4 in essentially the same way that IPC corresponds to modal logic S4. In Ardeshir [3] and Ardeshir and Ruitenburg [4] we introduce Visser algebras (where we named them basic algebras), which correspond to BPC in the same way that Heyting algebras correspond to IPC and that Boolean algebras correspond to classical propositional calculus (CPC). In Section 2 we present axiomatizations and some elementary properties of both BPC and Visser algebras.

The double negation construction of Boolean algebras from Heyting algebras is well known (see Balbes and Dwinger [6, Theorem IX.5.3] or Johnstone [7, p. 10]). It is natural to consider how closely one can repeat this construction over Visser algebras. Surprisingly the end result still works, although in details we use several new ideas.

Glivenko's well-established theorem (see [6, Section VIII.4 plus Theorem IX.5.3] or van Dalen [5, end of Section 5.2]) also goes through, but with an interesting reformulation. Given propositional formula $\psi$, define

$$
\xi(\psi):=((\top \rightarrow \psi) \rightarrow \psi) \rightarrow(\top \rightarrow \psi) .
$$

Received February 5, 2013; accepted October 9, 2013

First published online November 24, 2015

2010 Mathematics Subject Classification: Primary 03G05, 03G25, 06D20; Secondary 03B20

Keywords: Boolean algebra, Glivenko theorem, Visser logic

(C) 2016 by University of Notre Dame 10.1215/00294527-3339473 


\title{
Boolean Algebras in Visser Algebras
}

\author{
Majid Alizadeh, Mohammad Ardeshir, and Wim Ruitenburg
}

\begin{abstract}
We generalize the double negation construction of Boolean algebras in Heyting algebras to a double negation construction of the same in Visser algebras (also known as basic algebras). This result allows us to generalize Glivenko's theorem from intuitionistic propositional logic and Heyting algebras to Visser's basic propositional logic and Visser algebras.
\end{abstract}

\section{Introduction}

Basic propositional calculus (BPC), which was introduced by Albert Visser in [8], captures a sublogic of intuitionistic propositional calculus (IPC) which corresponds to modal logic K4 in essentially the same way that IPC corresponds to modal logic S4. In Ardeshir [3] and Ardeshir and Ruitenburg [4] we introduce Visser algebras (where we named them basic algebras), which correspond to BPC in the same way that Heyting algebras correspond to IPC and that Boolean algebras correspond to classical propositional calculus (CPC). In Section 2 we present axiomatizations and some elementary properties of both BPC and Visser algebras.

The double negation construction of Boolean algebras from Heyting algebras is well known (see Balbes and Dwinger [6, Theorem IX.5.3] or Johnstone [7, p. 10]). It is natural to consider how closely one can repeat this construction over Visser algebras. Surprisingly the end result still works, although in details we use several new ideas.

Glivenko's well-established theorem (see [6, Section VIII.4 plus Theorem IX.5.3] or van Dalen [5, end of Section 5.2]) also goes through, but with an interesting reformulation. Given propositional formula $\psi$, define

$$
\xi(\psi):=((\top \rightarrow \psi) \rightarrow \psi) \rightarrow(\top \rightarrow \psi) .
$$

Received February 5, 2013; accepted October 9, 2013

First published online November 24, 2015

2010 Mathematics Subject Classification: Primary 03G05, 03G25, 06D20; Secondary 03B20

Keywords: Boolean algebra, Glivenko theorem, Visser logic

(C) 2016 by University of Notre Dame 10.1215/00294527-3339473 


\title{
Boolean Algebras in Visser Algebras
}

\author{
Majid Alizadeh, Mohammad Ardeshir, and Wim Ruitenburg
}

\begin{abstract}
We generalize the double negation construction of Boolean algebras in Heyting algebras to a double negation construction of the same in Visser algebras (also known as basic algebras). This result allows us to generalize Glivenko's theorem from intuitionistic propositional logic and Heyting algebras to Visser's basic propositional logic and Visser algebras.
\end{abstract}

\section{Introduction}

Basic propositional calculus (BPC), which was introduced by Albert Visser in [8], captures a sublogic of intuitionistic propositional calculus (IPC) which corresponds to modal logic K4 in essentially the same way that IPC corresponds to modal logic S4. In Ardeshir [3] and Ardeshir and Ruitenburg [4] we introduce Visser algebras (where we named them basic algebras), which correspond to BPC in the same way that Heyting algebras correspond to IPC and that Boolean algebras correspond to classical propositional calculus (CPC). In Section 2 we present axiomatizations and some elementary properties of both BPC and Visser algebras.

The double negation construction of Boolean algebras from Heyting algebras is well known (see Balbes and Dwinger [6, Theorem IX.5.3] or Johnstone [7, p. 10]). It is natural to consider how closely one can repeat this construction over Visser algebras. Surprisingly the end result still works, although in details we use several new ideas.

Glivenko's well-established theorem (see [6, Section VIII.4 plus Theorem IX.5.3] or van Dalen [5, end of Section 5.2]) also goes through, but with an interesting reformulation. Given propositional formula $\psi$, define

$$
\xi(\psi):=((\top \rightarrow \psi) \rightarrow \psi) \rightarrow(\top \rightarrow \psi) .
$$

Received February 5, 2013; accepted October 9, 2013

First published online November 24, 2015

2010 Mathematics Subject Classification: Primary 03G05, 03G25, 06D20; Secondary 03B20

Keywords: Boolean algebra, Glivenko theorem, Visser logic

(C) 2016 by University of Notre Dame 10.1215/00294527-3339473 


\title{
Boolean Algebras in Visser Algebras
}

\author{
Majid Alizadeh, Mohammad Ardeshir, and Wim Ruitenburg
}

\begin{abstract}
We generalize the double negation construction of Boolean algebras in Heyting algebras to a double negation construction of the same in Visser algebras (also known as basic algebras). This result allows us to generalize Glivenko's theorem from intuitionistic propositional logic and Heyting algebras to Visser's basic propositional logic and Visser algebras.
\end{abstract}

\section{Introduction}

Basic propositional calculus (BPC), which was introduced by Albert Visser in [8], captures a sublogic of intuitionistic propositional calculus (IPC) which corresponds to modal logic K4 in essentially the same way that IPC corresponds to modal logic S4. In Ardeshir [3] and Ardeshir and Ruitenburg [4] we introduce Visser algebras (where we named them basic algebras), which correspond to BPC in the same way that Heyting algebras correspond to IPC and that Boolean algebras correspond to classical propositional calculus (CPC). In Section 2 we present axiomatizations and some elementary properties of both BPC and Visser algebras.

The double negation construction of Boolean algebras from Heyting algebras is well known (see Balbes and Dwinger [6, Theorem IX.5.3] or Johnstone [7, p. 10]). It is natural to consider how closely one can repeat this construction over Visser algebras. Surprisingly the end result still works, although in details we use several new ideas.

Glivenko's well-established theorem (see [6, Section VIII.4 plus Theorem IX.5.3] or van Dalen [5, end of Section 5.2]) also goes through, but with an interesting reformulation. Given propositional formula $\psi$, define

$$
\xi(\psi):=((\top \rightarrow \psi) \rightarrow \psi) \rightarrow(\top \rightarrow \psi) .
$$

Received February 5, 2013; accepted October 9, 2013

First published online November 24, 2015

2010 Mathematics Subject Classification: Primary 03G05, 03G25, 06D20; Secondary 03B20

Keywords: Boolean algebra, Glivenko theorem, Visser logic

(C) 2016 by University of Notre Dame 10.1215/00294527-3339473 


\title{
Boolean Algebras in Visser Algebras
}

\author{
Majid Alizadeh, Mohammad Ardeshir, and Wim Ruitenburg
}

\begin{abstract}
We generalize the double negation construction of Boolean algebras in Heyting algebras to a double negation construction of the same in Visser algebras (also known as basic algebras). This result allows us to generalize Glivenko's theorem from intuitionistic propositional logic and Heyting algebras to Visser's basic propositional logic and Visser algebras.
\end{abstract}

\section{Introduction}

Basic propositional calculus (BPC), which was introduced by Albert Visser in [8], captures a sublogic of intuitionistic propositional calculus (IPC) which corresponds to modal logic K4 in essentially the same way that IPC corresponds to modal logic S4. In Ardeshir [3] and Ardeshir and Ruitenburg [4] we introduce Visser algebras (where we named them basic algebras), which correspond to BPC in the same way that Heyting algebras correspond to IPC and that Boolean algebras correspond to classical propositional calculus (CPC). In Section 2 we present axiomatizations and some elementary properties of both BPC and Visser algebras.

The double negation construction of Boolean algebras from Heyting algebras is well known (see Balbes and Dwinger [6, Theorem IX.5.3] or Johnstone [7, p. 10]). It is natural to consider how closely one can repeat this construction over Visser algebras. Surprisingly the end result still works, although in details we use several new ideas.

Glivenko's well-established theorem (see [6, Section VIII.4 plus Theorem IX.5.3] or van Dalen [5, end of Section 5.2]) also goes through, but with an interesting reformulation. Given propositional formula $\psi$, define

$$
\xi(\psi):=((\top \rightarrow \psi) \rightarrow \psi) \rightarrow(\top \rightarrow \psi) .
$$

Received February 5, 2013; accepted October 9, 2013

First published online November 24, 2015

2010 Mathematics Subject Classification: Primary 03G05, 03G25, 06D20; Secondary 03B20

Keywords: Boolean algebra, Glivenko theorem, Visser logic

(C) 2016 by University of Notre Dame 10.1215/00294527-3339473 


\title{
Boolean Algebras in Visser Algebras
}

\author{
Majid Alizadeh, Mohammad Ardeshir, and Wim Ruitenburg
}

\begin{abstract}
We generalize the double negation construction of Boolean algebras in Heyting algebras to a double negation construction of the same in Visser algebras (also known as basic algebras). This result allows us to generalize Glivenko's theorem from intuitionistic propositional logic and Heyting algebras to Visser's basic propositional logic and Visser algebras.
\end{abstract}

\section{Introduction}

Basic propositional calculus (BPC), which was introduced by Albert Visser in [8], captures a sublogic of intuitionistic propositional calculus (IPC) which corresponds to modal logic K4 in essentially the same way that IPC corresponds to modal logic S4. In Ardeshir [3] and Ardeshir and Ruitenburg [4] we introduce Visser algebras (where we named them basic algebras), which correspond to BPC in the same way that Heyting algebras correspond to IPC and that Boolean algebras correspond to classical propositional calculus (CPC). In Section 2 we present axiomatizations and some elementary properties of both BPC and Visser algebras.

The double negation construction of Boolean algebras from Heyting algebras is well known (see Balbes and Dwinger [6, Theorem IX.5.3] or Johnstone [7, p. 10]). It is natural to consider how closely one can repeat this construction over Visser algebras. Surprisingly the end result still works, although in details we use several new ideas.

Glivenko's well-established theorem (see [6, Section VIII.4 plus Theorem IX.5.3] or van Dalen [5, end of Section 5.2]) also goes through, but with an interesting reformulation. Given propositional formula $\psi$, define

$$
\xi(\psi):=((\top \rightarrow \psi) \rightarrow \psi) \rightarrow(\top \rightarrow \psi) .
$$

Received February 5, 2013; accepted October 9, 2013

First published online November 24, 2015

2010 Mathematics Subject Classification: Primary 03G05, 03G25, 06D20; Secondary 03B20

Keywords: Boolean algebra, Glivenko theorem, Visser logic

(C) 2016 by University of Notre Dame 10.1215/00294527-3339473 


\title{
Boolean Algebras in Visser Algebras
}

\author{
Majid Alizadeh, Mohammad Ardeshir, and Wim Ruitenburg
}

\begin{abstract}
We generalize the double negation construction of Boolean algebras in Heyting algebras to a double negation construction of the same in Visser algebras (also known as basic algebras). This result allows us to generalize Glivenko's theorem from intuitionistic propositional logic and Heyting algebras to Visser's basic propositional logic and Visser algebras.
\end{abstract}

\section{Introduction}

Basic propositional calculus (BPC), which was introduced by Albert Visser in [8], captures a sublogic of intuitionistic propositional calculus (IPC) which corresponds to modal logic K4 in essentially the same way that IPC corresponds to modal logic S4. In Ardeshir [3] and Ardeshir and Ruitenburg [4] we introduce Visser algebras (where we named them basic algebras), which correspond to BPC in the same way that Heyting algebras correspond to IPC and that Boolean algebras correspond to classical propositional calculus (CPC). In Section 2 we present axiomatizations and some elementary properties of both BPC and Visser algebras.

The double negation construction of Boolean algebras from Heyting algebras is well known (see Balbes and Dwinger [6, Theorem IX.5.3] or Johnstone [7, p. 10]). It is natural to consider how closely one can repeat this construction over Visser algebras. Surprisingly the end result still works, although in details we use several new ideas.

Glivenko's well-established theorem (see [6, Section VIII.4 plus Theorem IX.5.3] or van Dalen [5, end of Section 5.2]) also goes through, but with an interesting reformulation. Given propositional formula $\psi$, define

$$
\xi(\psi):=((\top \rightarrow \psi) \rightarrow \psi) \rightarrow(\top \rightarrow \psi) .
$$

Received February 5, 2013; accepted October 9, 2013

First published online November 24, 2015

2010 Mathematics Subject Classification: Primary 03G05, 03G25, 06D20; Secondary 03B20

Keywords: Boolean algebra, Glivenko theorem, Visser logic

(C) 2016 by University of Notre Dame 10.1215/00294527-3339473 


\title{
Boolean Algebras in Visser Algebras
}

\author{
Majid Alizadeh, Mohammad Ardeshir, and Wim Ruitenburg
}

\begin{abstract}
We generalize the double negation construction of Boolean algebras in Heyting algebras to a double negation construction of the same in Visser algebras (also known as basic algebras). This result allows us to generalize Glivenko's theorem from intuitionistic propositional logic and Heyting algebras to Visser's basic propositional logic and Visser algebras.
\end{abstract}

\section{Introduction}

Basic propositional calculus (BPC), which was introduced by Albert Visser in [8], captures a sublogic of intuitionistic propositional calculus (IPC) which corresponds to modal logic K4 in essentially the same way that IPC corresponds to modal logic S4. In Ardeshir [3] and Ardeshir and Ruitenburg [4] we introduce Visser algebras (where we named them basic algebras), which correspond to BPC in the same way that Heyting algebras correspond to IPC and that Boolean algebras correspond to classical propositional calculus (CPC). In Section 2 we present axiomatizations and some elementary properties of both BPC and Visser algebras.

The double negation construction of Boolean algebras from Heyting algebras is well known (see Balbes and Dwinger [6, Theorem IX.5.3] or Johnstone [7, p. 10]). It is natural to consider how closely one can repeat this construction over Visser algebras. Surprisingly the end result still works, although in details we use several new ideas.

Glivenko's well-established theorem (see [6, Section VIII.4 plus Theorem IX.5.3] or van Dalen [5, end of Section 5.2]) also goes through, but with an interesting reformulation. Given propositional formula $\psi$, define

$$
\xi(\psi):=((\top \rightarrow \psi) \rightarrow \psi) \rightarrow(\top \rightarrow \psi) .
$$

Received February 5, 2013; accepted October 9, 2013

First published online November 24, 2015

2010 Mathematics Subject Classification: Primary 03G05, 03G25, 06D20; Secondary 03B20

Keywords: Boolean algebra, Glivenko theorem, Visser logic

(C) 2016 by University of Notre Dame 10.1215/00294527-3339473 


\title{
Boolean Algebras in Visser Algebras
}

\author{
Majid Alizadeh, Mohammad Ardeshir, and Wim Ruitenburg
}

\begin{abstract}
We generalize the double negation construction of Boolean algebras in Heyting algebras to a double negation construction of the same in Visser algebras (also known as basic algebras). This result allows us to generalize Glivenko's theorem from intuitionistic propositional logic and Heyting algebras to Visser's basic propositional logic and Visser algebras.
\end{abstract}

\section{Introduction}

Basic propositional calculus (BPC), which was introduced by Albert Visser in [8], captures a sublogic of intuitionistic propositional calculus (IPC) which corresponds to modal logic K4 in essentially the same way that IPC corresponds to modal logic S4. In Ardeshir [3] and Ardeshir and Ruitenburg [4] we introduce Visser algebras (where we named them basic algebras), which correspond to BPC in the same way that Heyting algebras correspond to IPC and that Boolean algebras correspond to classical propositional calculus (CPC). In Section 2 we present axiomatizations and some elementary properties of both BPC and Visser algebras.

The double negation construction of Boolean algebras from Heyting algebras is well known (see Balbes and Dwinger [6, Theorem IX.5.3] or Johnstone [7, p. 10]). It is natural to consider how closely one can repeat this construction over Visser algebras. Surprisingly the end result still works, although in details we use several new ideas.

Glivenko's well-established theorem (see [6, Section VIII.4 plus Theorem IX.5.3] or van Dalen [5, end of Section 5.2]) also goes through, but with an interesting reformulation. Given propositional formula $\psi$, define

$$
\xi(\psi):=((\top \rightarrow \psi) \rightarrow \psi) \rightarrow(\top \rightarrow \psi) .
$$

Received February 5, 2013; accepted October 9, 2013

First published online November 24, 2015

2010 Mathematics Subject Classification: Primary 03G05, 03G25, 06D20; Secondary 03B20

Keywords: Boolean algebra, Glivenko theorem, Visser logic

(C) 2016 by University of Notre Dame 10.1215/00294527-3339473 


\title{
Boolean Algebras in Visser Algebras
}

\author{
Majid Alizadeh, Mohammad Ardeshir, and Wim Ruitenburg
}

\begin{abstract}
We generalize the double negation construction of Boolean algebras in Heyting algebras to a double negation construction of the same in Visser algebras (also known as basic algebras). This result allows us to generalize Glivenko's theorem from intuitionistic propositional logic and Heyting algebras to Visser's basic propositional logic and Visser algebras.
\end{abstract}

\section{Introduction}

Basic propositional calculus (BPC), which was introduced by Albert Visser in [8], captures a sublogic of intuitionistic propositional calculus (IPC) which corresponds to modal logic K4 in essentially the same way that IPC corresponds to modal logic S4. In Ardeshir [3] and Ardeshir and Ruitenburg [4] we introduce Visser algebras (where we named them basic algebras), which correspond to BPC in the same way that Heyting algebras correspond to IPC and that Boolean algebras correspond to classical propositional calculus (CPC). In Section 2 we present axiomatizations and some elementary properties of both BPC and Visser algebras.

The double negation construction of Boolean algebras from Heyting algebras is well known (see Balbes and Dwinger [6, Theorem IX.5.3] or Johnstone [7, p. 10]). It is natural to consider how closely one can repeat this construction over Visser algebras. Surprisingly the end result still works, although in details we use several new ideas.

Glivenko's well-established theorem (see [6, Section VIII.4 plus Theorem IX.5.3] or van Dalen [5, end of Section 5.2]) also goes through, but with an interesting reformulation. Given propositional formula $\psi$, define

$$
\xi(\psi):=((\top \rightarrow \psi) \rightarrow \psi) \rightarrow(\top \rightarrow \psi) .
$$

Received February 5, 2013; accepted October 9, 2013

First published online November 24, 2015

2010 Mathematics Subject Classification: Primary 03G05, 03G25, 06D20; Secondary 03B20

Keywords: Boolean algebra, Glivenko theorem, Visser logic

(C) 2016 by University of Notre Dame 10.1215/00294527-3339473 